\title{
Entropy generation and unified optimization of Carnot-like and low-dissipation refrigerators
}

\author{
Julian Gonzalez-Ayala ${ }^{1}$, A. Medina ${ }^{1}$, J. M. M. Roco ${ }^{1,2}$ and A. Calvo Hernández ${ }^{1,2}$ \\ ${ }^{1}$ Departamento de Física Aplicada and ${ }^{2}$ Instituto Universitario de Física Fundamental y Matemáticas (IUFFyM) \\ Universidad de Salamanca, 37008 Salamanca, Spain
}

\begin{abstract}
The connection between Carnot-like and low-dissipation refrigerators is proposed by means of their entropy generation and the optimization of two unified, compromise-based figures of merit. Their optimization shows that only a limited set of heat transfer laws in the Carnot-like model are compatible with the results stemming from the low-dissipation approximation, even though there is an agreement of the related physical spaces of variables. A comparison between two operation regimes and relations among entropy generation, efficiency, cooling-power and power-input are obtained, with emphasis on the role of dissipation symmetries. The results extend previous findings for heat engines at maximum power conditions.
\end{abstract}

\section{INTRODUCTION}

The pursue of energy converter models to describe more accurately efficient real-life devices, and refrigerators in particular, has brought a variety of models and optimization criteria according to the different nature and scale range: from quantum [1-11] to macroscopic [12-19] passing through mesoscopic [20-29] levels and including solar-assisted cooling techniques [30, 31].

Most models are based on finite-time thermodynamics considerations [32, 33]. Among them, but very different in nature are the Carnot-like (CL) [13-18, 34] and the low-dissipation (LD)[35-39] models whose predicted results are quite close to the region of experimental data [12]. The key peculiarities of each model can be summarized as follow. The CL engine makes use of thermal conductances and heat transfer laws to model the heat fluxes and entropy production, this gives information about the heat-exchanges nature and thermal properties of the material involved in the device. The LD model, on the other hand, allows obtaining upper and lower performance bounds under certain operation regime depending only on the dissipations' symmetries, accounted by an specific entropy generation without any information about the heat-fluxes nature. The optimization variables are, in the CL case, the working substance temperatures at the isothermal processes, while in the LD case are the contact-times at the isothermal processes. The generality of the LD model reproduces behaviors of both endoreversible and irreversible engines, depending on timeconstraints [40]. For the CL engine the difference between both behaviors is the presence of a heat-leak. This arise the question of whether a correspondence between these models can be established, and if so, to explain the role of the heat-leak in time-constraints.

In recent years, the LD heat-engine (HE) perspectives have increased with its applicability to models that incorporate fluctuations in microscopic and quantum systems [39, 41-43]. Their relevance in microscopic refrigeratorengines (RE's), whose studies are mostly based in specific heat transfer mechanisms, have not been widely explored, making the unified study of HE's and RE's energetics an on-going task.

In a recent paper [44] the connection between the CL and the LD models was proposed for HE's at maximum power, showing that the set of variables that describe each model can be related through the entropy generation. The particular case of an inverse-of-temperature heat law has been recently addressed in [45]. The physical space of parameters is equivalent in the two descriptions, however, maximum-power efficiencies do not match exactly for arbitrary heat transfer laws. This could underlay in the nature of each approach: the LD model based on a specific entropy-generation law and the CL model over the heat fluxes. Nevertheless, for a range of heat transfer laws the correspondence between these models is reasonably good.

Beyond models, another important point is the proposal of unified figures of merit for any kind of energy converter [19, 39, 46-51]. In particular, we consider in this paper two optimization criteria: the so called $\chi[36]$ and $\Omega$ [52] criteria, whose validity for both RE's and HE's has been widely acknowledged [53-58].

To highlight the resemblance between the treatment of HE's and RE's we present a parallel description to that appearing in [44] for HE's with special emphasis on the role played by the heat-leaks and contact-times. We will present how-well the efficiencies from the CL RE (for a wide family of heat transfer laws) fit the LD assumption.

The article is organized as follows: In Section 2 we present the unified criteria $\chi$ and $\Omega$. In Section 3 the mathematical correspondence among the characteristics variables of both models for RE's is proposed. In Sections 4 and 5 , respectively, we analyze the maximum- $\chi$ and $\Omega$ regimes in both models; in Section 6 we present a comparison of the some relevant energetic magnitudes, including the coefficient of performance (COP) and entropy generation within the LD model framework. Finally, some concluding remarks are presented in Section 7 . 
(a)

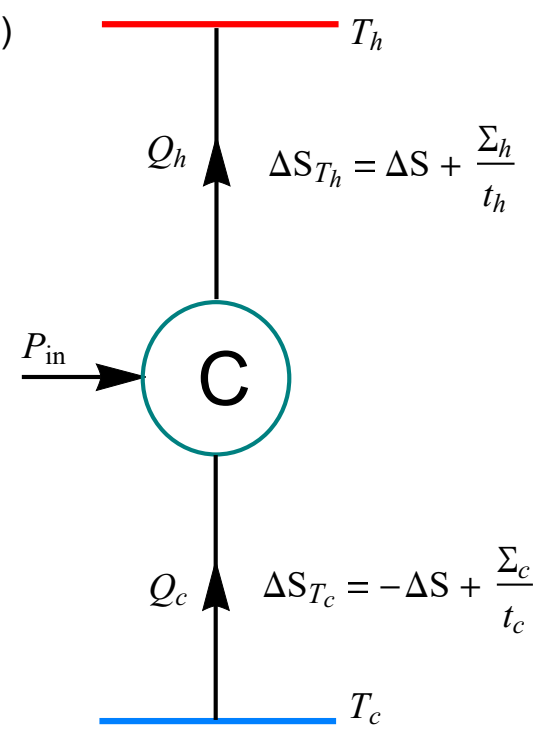

(b)

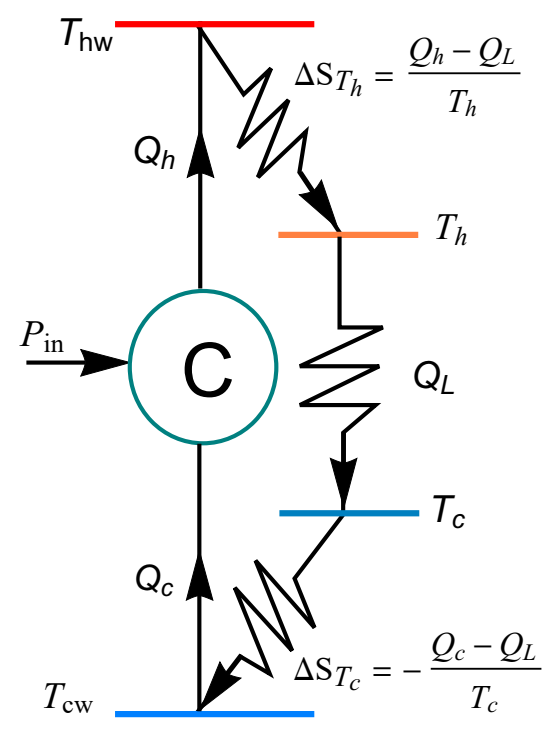

FIG. 1. Sketchs of a LD refrigeration (a) and of an irreversible Carnot-like RE with a heat-leak $Q_{L}(\mathbf{b})$.

\section{UNIFIED FIGURES OF MERIT $\chi$ AND $\Omega$ FOR HE'S AND RE'S}

The function $\chi$ is defined as the efficiency of the engine times the heat-flux entering into the working substance in a cycle period, that is,

$$
\chi=\frac{z Q_{i n}}{t},
$$

being $z=\eta(\epsilon)$ the efficiency of the energy converter and $Q_{i n}=Q_{\mathrm{h}}\left(Q_{\mathrm{c}}\right)$ for HE's (RE's), then

$$
\begin{aligned}
& \chi^{(H E)}=\frac{\eta Q_{\mathrm{h}}}{t}=P, \\
& \chi^{(R E)}=\frac{\epsilon Q_{\mathrm{c}}}{t}=\epsilon R,
\end{aligned}
$$

where $P=\left(Q_{\mathrm{h}}-Q_{\mathrm{c}}\right) / t$, is the power-output of the HE and $R=Q_{\mathrm{c}} / t$ is the cooling-power of the RE. For $\mathrm{RE}$ the role of the efficiency is taken by the COP, $\epsilon$, and the analogue of Carnot efficiency $\eta_{C}=1-\tau$ and CurzonAhlborn (CA) efficiency $\eta_{C A}=1-\sqrt{1-\eta_{C}}$ is played by $\epsilon_{C}=\tau /(1-\tau)$ and $\epsilon_{C A}=\sqrt{1+\epsilon_{C}}-1$ [36], where $\tau=T_{\mathrm{c}} / T_{\mathrm{h}}$ being the cold-to-hot bath temperature ratio.

In the line of unified studies of HE's and RE's a relevant role is played by the so-called $\Omega$ function. This ecological-like figure of merit takes into account the unavoidable losses caused by the irreversible nature of the finite-time periodic processes and is defined as follows [53]

$$
\Omega \equiv\left(2 z-z_{\max }\right) \frac{E_{i n}}{t},
$$

where $E_{i n}$ is the heat input for HE's and the work input $W_{\text {in }}$ for RE's. Then,

$$
\begin{aligned}
& \Omega^{H E}=\left(2 \eta-\eta_{\max }\right) \frac{Q_{\mathrm{h}}}{t}, \\
& \Omega^{R E}=\left(2 \epsilon-\epsilon_{\max }\right) P_{i n},
\end{aligned}
$$

where $P_{i n}=\left(Q_{\mathrm{h}}-Q_{\mathrm{c}}\right) / t$.

\section{CORRESPONDENCE BETWEEN THE RE'S VARIABLES OF BOTH MODELS}

The LD model for RE considers a base-line Carnot refrigerator working between the temperatures $T_{\mathrm{c}}$ and $T_{\mathrm{h}}>T_{\mathrm{c}}$ (see Fig. 1a). A deviation from the reversible scenario is modeled by additive terms in the entropy changes at the heat reservoirs, given by [53]

$$
\begin{aligned}
& \Delta S_{T_{\mathrm{h}}}=\Delta S+\frac{\Sigma_{\mathrm{h}}}{t_{\mathrm{h}}}, \\
& \Delta S_{T_{\mathrm{c}}}=-\Delta S+\frac{\Sigma_{\mathrm{c}}}{t_{\mathrm{c}}},
\end{aligned}
$$

where $\Sigma_{\mathrm{h}}$ and $\Sigma_{\mathrm{c}}$ are some dissipative coefficients that contain all the information of intrinsic dissipative deviceproperties; $t_{\mathrm{h}}$ and $t_{\mathrm{c}}$ are the contact-times with the hot and cold reservoirs. We assume that the adiabatic processes time can be neglected. $\Delta S$ is the entropy change of the working fluid in the cold isothermal process. The total entropy change is

$$
\Delta S_{\mathrm{tot}}=\frac{\Delta S_{T_{\mathrm{h}}}}{T_{\mathrm{h}}}+\frac{\Delta S_{T_{\mathrm{c}}}}{T_{\mathrm{c}}}=\frac{\Sigma_{\mathrm{h}}}{t_{\mathrm{h}}}+\frac{\Sigma_{\mathrm{c}}}{t_{\mathrm{c}}} \geq 0 .
$$

where the reversible scenario $\left(\Delta S_{\text {tot }}=0\right)$ is achieved in the limits $t_{\mathrm{h}} \rightarrow \infty$ and $t_{\mathrm{c}} \rightarrow \infty$.

By means of the dimensionless variables defined in [60] that take into account the size of the system: $\alpha \equiv t_{\mathrm{c}} / t$, $\widetilde{\Sigma}_{\mathrm{c}} \equiv \Sigma_{\mathrm{c}} / \Sigma_{\mathrm{T}}$ and $\widetilde{t} \equiv(t \Delta S) / \Sigma_{\mathrm{T}}$, where $t=t_{\mathrm{h}}+t_{\mathrm{c}}$ and $\Sigma_{\mathrm{T}} \equiv \Sigma_{\mathrm{h}}+\Sigma_{\mathrm{c}}$; we define a characteristic total entropy 
production per unit time

$$
\dot{\overline{\Delta S}}_{\text {tot }} \equiv \frac{\Delta S_{t o t}}{\widetilde{t} \Delta S}=\frac{\Delta S_{\text {tot }}}{t} \frac{\Sigma_{\mathrm{T}}}{\Delta S^{2}}=\frac{1}{\widetilde{t}}\left[\frac{1-\widetilde{\Sigma}_{\mathrm{c}}}{(1-\alpha) \widetilde{t}}+\frac{\widetilde{\Sigma}_{\mathrm{c}}}{\alpha \widetilde{t}}\right] .
$$

In the CL-RE the entropy change of the internal reversible cycle is zero and the total entropy production is that generated at the couplings with the external heat reservoirs as depicted in Fig. 1b. According to this figure $Q_{\mathrm{h}}=T_{\mathrm{hw}} \Delta S \geq 0$ and $Q_{\mathrm{c}}=T_{\mathrm{cw}} \Delta S \geq 0$, where $\Delta S$ is the entropy generation at the heat reservoir $T_{\mathrm{cw}}$, and $Q_{\mathrm{L}} \geq 0$ is a heat-leak between the reservoirs $T_{\mathrm{h}}$ and $T_{\mathrm{c}}$, then

$$
\begin{aligned}
& \Delta S_{T_{\mathrm{h}}}=\frac{Q_{\mathrm{h}}}{T_{\mathrm{h}}}-\frac{Q_{L}}{T_{\mathrm{h}}}=\Delta S+\left(-1+a_{\mathrm{h}}^{-1}-\tau \widetilde{Q}_{L}\right) \Delta S, \\
& \Delta S_{T_{\mathrm{c}}}=-\frac{Q_{\mathrm{c}}}{T_{\mathrm{c}}}+\frac{Q_{L}}{T_{\mathrm{c}}}=-\Delta S+\left(-a_{\mathrm{c}}+1+\widetilde{Q}_{L}\right) \Delta S,
\end{aligned}
$$

where $a_{\mathrm{h}}=T_{\mathrm{h}} / T_{\mathrm{hw}} \leq 1, a_{\mathrm{c}}=T_{\mathrm{cw}} / T_{\mathrm{c}} \leq 1$ and a characteristic heat-leak is defined as $\widetilde{Q}_{L} \equiv Q_{L} /\left(T_{\mathrm{c}} \Delta S\right)$. A comparison between Eqs. (7) and (8) gives the expressions associated to $\Sigma_{\mathrm{h}}$ and $\Sigma_{\mathrm{c}}$,

$$
\begin{aligned}
& \frac{\Sigma_{\mathrm{h}}}{t_{\mathrm{h}}}=\left(-1+a_{\mathrm{h}}^{-1}-\tau \widetilde{Q}_{L}\right) \Delta S \\
& \frac{\Sigma_{\mathrm{c}}}{t_{\mathrm{c}}}=\left(-a_{\mathrm{c}}+1+\widetilde{Q}_{L}\right) \Delta S .
\end{aligned}
$$

The heat-leak is not a feature appearing in the LD model, nevertheless (as will be shown later) it's possible to link it within the effects of the dissipation term $T_{\mathrm{c}} \widetilde{\Sigma}_{\mathrm{c}} / t_{\mathrm{c}}$. From the above two equations it is easy to obtain the following relations between the variables of the LD and CL models,

$$
\begin{aligned}
\widetilde{\Sigma}_{\mathrm{c}}^{-1} & =1+\left(\frac{1-\alpha}{\alpha}\right)\left(\frac{-1+a_{\mathrm{h}}^{-1}-\tau \widetilde{Q}_{\mathrm{L}}}{-a_{\mathrm{c}}+1+\widetilde{Q}_{\mathrm{L}}}\right), \\
\widetilde{t} & =\frac{1}{\alpha\left(-a_{\mathrm{c}}+1+\widetilde{Q}_{\mathrm{L}}\right)\left[1+\left(\frac{1-\alpha}{\alpha}\right)\left(\frac{-1+a_{\mathrm{h}}^{-1}-\tau \widetilde{Q}_{\mathrm{L}}}{-a_{\mathrm{c}}+1+\widetilde{Q}_{\mathrm{L}}}\right)\right]},
\end{aligned}
$$

which can be summarized as:

$$
\frac{\widetilde{\Sigma}_{\mathrm{c}}}{\alpha \widetilde{t}}=-a_{\mathrm{c}}+1+\widetilde{Q}_{\mathrm{L}}
$$

This equation allows for a consistent, thermodynamics interpretation of the heat-leak in the LD model. It can be rewritten as

$$
Q_{\mathrm{L}}=T_{\mathrm{c}} \frac{\Sigma_{\mathrm{c}}}{t_{\mathrm{c}}}-\left(T_{\mathrm{c}}-T_{\mathrm{cw}}\right) \Delta S,
$$

where the first term of the right-hand-side is the heat dissipated to the cold reservoir in the LD model $\left(Q_{\mathrm{c}, \text { diss }}^{L D}\right)$. Since $\Delta S$ is the working-substance entropy change while in contact with the $T_{\mathrm{cw}}$ heat reservoir, then, $T_{\mathrm{c}} \Delta S$ corresponds to the heat exchanged when $T_{\mathrm{cw}}=T_{\mathrm{c}}$ and $\left(T_{\mathrm{c}}-T_{\mathrm{cw}}\right) \Delta S$ is the difference between the heat-input at the totally reversible situation $\left(Q_{c, \text { rev }}\right)$ and that of nonequilibrium $\left(Q_{\mathrm{c}, \text { neq }}\right)$. We name this quantity $Q_{\mathrm{c}, \text { loss }}^{\text {endo }} \equiv$ $Q_{c, \text { rev }}-Q_{\mathrm{c} \text {, neq }}$, a heat-input loss due to the endoreversibility of the CL engine. In this way, Eq. (16) is

$$
Q_{\mathrm{L}}=Q_{\mathrm{c}, \text { diss }}^{L D}-Q_{\mathrm{c}, \text { loss }}^{\text {endo }}
$$

Thus, the heat-leak is the part of the dissipated heat to the cold reservoir that has not an endoreversible origin in the CL model. The term $Q_{c \text {, loss }}^{\text {endo }}$ is very similar to the one obtained in the so-called "geometric dissipation" [61], where a dissipation-like term can be attached to a reversible cycle if one subtracts the heat released by a Carnot cycle from the heat released by the reversible cycle, when both cycles operate between the same heat reservoirs and with the same heat input.

Regarding the physical region for the RE's it can be deduced from the Clausius inequality in Eq. (9). Thus we require that for any $a_{\mathrm{c}, \mathrm{h}}$ and $\widetilde{Q}_{\mathrm{L}}$ values $-\Delta S_{T_{\mathrm{c}}} \geq 0$ and $\Delta S_{T_{\mathrm{h}}} \geq 0$ (Eqs. (11) and (12), respectively). This yields to $a_{\mathrm{h}}^{-1} \geq \tau \widetilde{Q}_{\mathrm{L}}$ and $a_{\mathrm{c}} \geq \widetilde{Q}_{\mathrm{L}}$ and since the latter condition is achieved first it defines the physical constraint $\widetilde{Q}_{\mathrm{L}} \in$ $\left[0, a_{\mathrm{c}}\right]$. This heat-leak constraint (from CL model-based arguments) applied to Eq. (16) gives a restriction upon the $\mathrm{LD}$ RE variables

$$
\widetilde{\Sigma}_{\mathrm{c}} \leq \alpha \widetilde{t}
$$

Later on, we will show that this is equivalent to requiring $\tilde{\chi} \geq 0$ (with only LD model considerations), showing that both physical spaces of variables are in agreement.

The correspondence among the variables of both models (Eq. (16)) is independent of heat transfer laws and operation regime. Despite of this, it is possible to see the influence of the heat-leak on $T_{\mathrm{cw}}$ and $T_{\mathrm{hw}}$ (which usually requires an explicit heat transfer law) and on the total operation time as can be seen in Fig. $2 \mathrm{a}$ and $2 \mathrm{~b}$, respectively.

In Fig. 2a the temperatures $T_{\mathrm{c}}, T_{\mathrm{cw}}, T_{\mathrm{hw}}$ and $T_{\mathrm{h}}$ are displayed as function of $a_{\mathrm{c}}$ and $\widetilde{Q}_{\mathrm{L}}$. For the CL RE it is well-known that thermal equilibrium between internal and external reservoirs (reversible situation) is achieved only if $\widetilde{Q}_{L}=0$. As the heat-leak increases the temperature $T_{\mathrm{hw}}$ departs from $T_{\mathrm{h}}$, producing a larger thermal gradients, and preventing thermal equilibrium. On the other hand, for the LD RE, $\widetilde{t}$ as a function of $a_{\mathrm{c}}$ and $\widetilde{Q}_{\mathrm{L}}$ (see Eq. (16)) is depicted in Fig. 2b, showing that as the heat-leak increases the total-time decreases and only when $a_{\mathrm{c}} \rightarrow 1$ and $\widetilde{Q}_{L} \rightarrow 0$ the reversible limit $t \rightarrow \infty$ can be achieved (if additionally $a_{\mathrm{h}} \rightarrow 1$ ). Fig. 2, where physical constraints are considered, displays a similar behavior of that reported for HE's (see Fig. 2 of ref. [44]). 

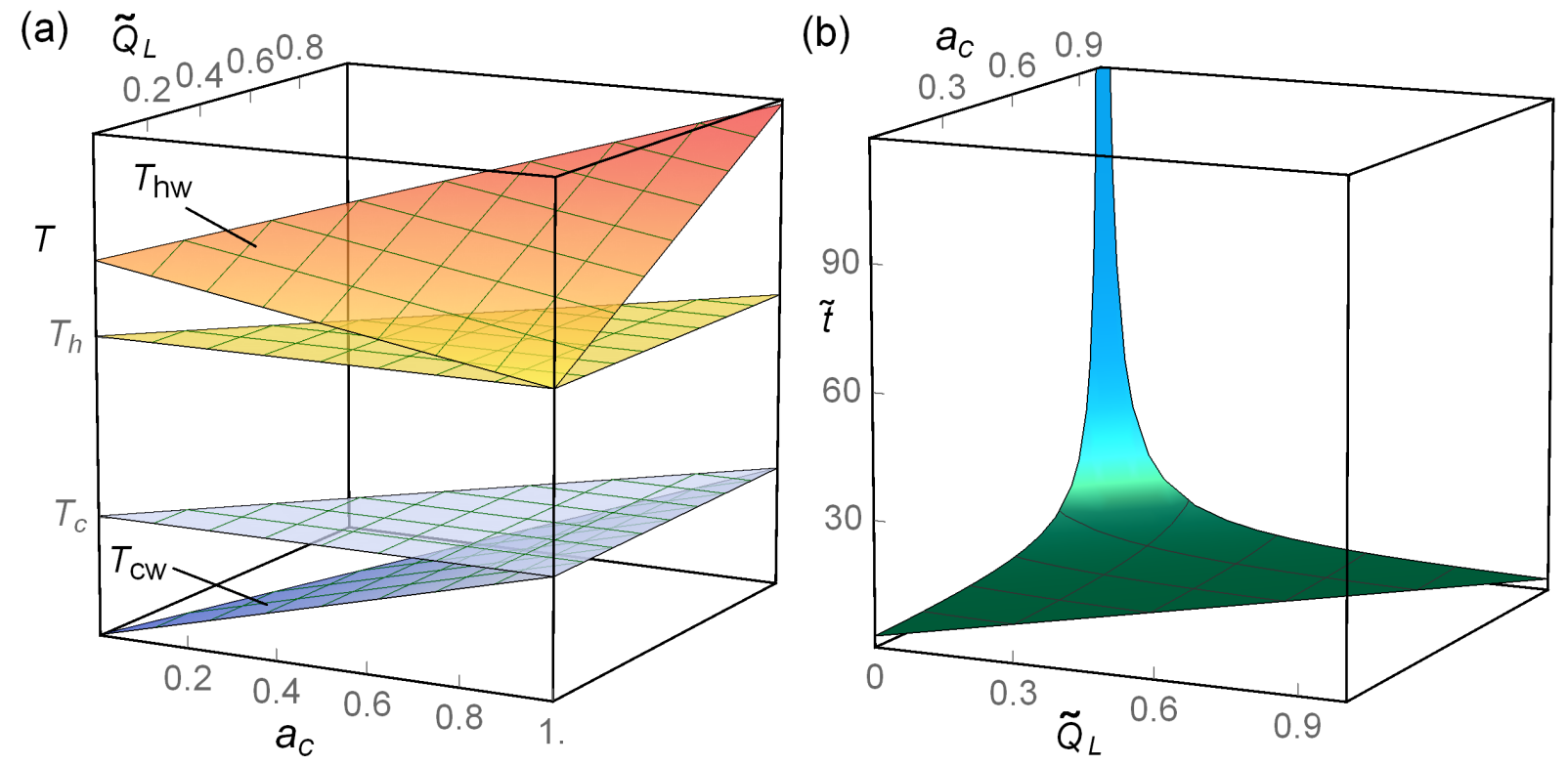

FIG. 2. a) $T_{\mathrm{hw}}$ and $T_{\mathrm{cw}}$ from Eq. (15). Note that as heat-leak appears both internal temperatures cannot be in thermal equilibrium with the external reservoirs. b) Total operation time $\widetilde{t}\left(\widetilde{Q}_{L}, a_{\mathrm{c}}\right)$ according to Eq. (16). In both figures the representative values $\alpha=\frac{1}{5}, \tau=0.4$ and $\widetilde{\Sigma}_{\mathrm{c}}=\frac{1}{2}$ are considered, the qualitative behavior for other values is the same.

\section{MAXIMUM- $\chi$ REGIME}

\section{A. Low dissipation refrigerator engine}

The input and output heats, (see Fig. 1a), are given by $[60]$

$$
\begin{aligned}
& \dot{\widetilde{Q}}_{\mathrm{h}} \equiv \frac{\widetilde{Q}_{\mathrm{h}}}{\widetilde{t}}=\frac{Q_{\mathrm{h}}}{T_{\mathrm{c}} \Delta S} \frac{\Sigma_{\mathrm{T}}}{t \Delta S}=\left(-1-\frac{1-\widetilde{\Sigma}_{\mathrm{c}}}{(1-\alpha) \widetilde{t}}\right) \frac{1}{\widetilde{t}} \\
& \dot{\widetilde{Q}}_{\mathrm{c}} \equiv \frac{\widetilde{Q}_{\mathrm{c}}}{\widetilde{t}}=\frac{Q_{\mathrm{c}}}{T_{\mathrm{c}} \Delta S} \frac{\Sigma_{\mathrm{T}}}{t \Delta S}=\left(1-\frac{\widetilde{\Sigma}_{\mathrm{c}}}{\alpha \widetilde{t}}\right) \frac{\tau}{\widetilde{t}}=\widetilde{R}
\end{aligned}
$$

the corresponding dimensionless power input $\widetilde{P}_{i n} \equiv$ $P_{i n} /\left(T_{\mathrm{c}} \Delta S \widetilde{t}\right)=\dot{\widetilde{Q}}_{\mathrm{h}}-\dot{\widetilde{Q}}_{\mathrm{c}}$ is

$$
\widetilde{P}_{i n}=\left(1-\tau+\frac{1-\widetilde{\Sigma}_{\mathrm{c}}}{(1-\alpha) \widetilde{t}}+\frac{\tau \widetilde{\Sigma_{c}}}{\alpha \widetilde{t}}\right) \frac{1}{\widetilde{t}}
$$

thus, $\widetilde{\epsilon}$ and $\tilde{\chi}$ (see Eq. 3) are given by

$$
\begin{aligned}
& \widetilde{\epsilon} \equiv \frac{\widetilde{R}}{\widetilde{P}_{i n}}=\frac{Q_{\mathrm{c}}}{W}=\epsilon=\frac{\left(1-\frac{\widetilde{\Sigma}_{\mathrm{c}}}{\alpha \widetilde{t}}\right) \tau}{1-\tau+\frac{1-\widetilde{\Sigma}_{\mathrm{c}}}{(1-\alpha) \tilde{t}}+\frac{\tau \widetilde{\Sigma}_{\mathrm{c}}}{\alpha \widetilde{t}}} . \\
& \tilde{\chi} \equiv \frac{\epsilon \widetilde{Q}_{\mathrm{c}}}{\widetilde{t}}=\frac{\epsilon Q_{\mathrm{c}}}{T_{\mathrm{c}} \Delta S} \frac{\Sigma_{\mathrm{T}}}{t \Delta S}=\frac{\left(1-\frac{\widetilde{\Sigma}_{\mathrm{c}}}{\alpha \widetilde{t}}\right)^{2} \frac{\tau^{2}}{\widetilde{t}}}{1-\tau+\frac{1-\widetilde{\Sigma}_{\mathrm{c}}}{(1-\alpha) \bar{t}}+\frac{\tau \widetilde{\Sigma}_{\mathrm{c}}}{\alpha \widetilde{t}}},
\end{aligned}
$$

Note that from Eqs. (23) and $(24) \epsilon$ and $\widetilde{\chi}$ are positive if $\widetilde{\Sigma}_{\mathrm{c}} \leq \alpha \widetilde{t}$, which is exactly the constraint appearing in Eq. (19) from heat-leak arguments. The maximization is achieved through $\alpha$ and $\tilde{t}$ by solving the system $\left(\frac{\partial \tilde{\chi}}{\partial \widetilde{t}}\right)_{\alpha}=0$ and $\left(\frac{\partial \tilde{\chi}}{\partial \alpha}\right)_{\widetilde{t}}=0$. The first condition leads to the optimum value $\widetilde{t^{*}}$

$$
\widetilde{t}^{*}=\frac{\widetilde{\Sigma}_{\mathrm{c}}}{2 \alpha}\left(3+\sqrt{\frac{9-\tau+8\left(\frac{\alpha}{1-\alpha}\right)\left(\frac{1-\widetilde{\Sigma}_{\mathrm{c}}}{\widetilde{\Sigma}_{\mathrm{c}}}\right)}{1-\tau}}\right)
$$

and the further maximization of $\tilde{\chi}^{*}\left(\alpha ; \widetilde{\Sigma}_{\mathrm{c}}, \tau\right)=$ $\widetilde{\chi}\left(\alpha, \widetilde{t}^{*} ; \widetilde{\Sigma}_{\mathrm{c}}, \tau\right)$ with respect to $\alpha$ is made numerically. The lower and upper bounds for $\epsilon_{\tilde{\chi}_{\max }}$ are

$$
\epsilon_{\tilde{\chi}_{\max }}^{-}=0 \leq \epsilon_{\widetilde{\chi}_{\max }} \leq \frac{\sqrt{9+8 \epsilon_{C}}-3}{2}=\epsilon_{\tilde{\chi}_{\max }}^{+}
$$

corresponding to the $\widetilde{\Sigma}_{\mathrm{c}}=0$ and $\widetilde{\Sigma}_{\mathrm{c}}=1$, respectively. The symmetric dissipation $\left(\widetilde{\Sigma}_{\mathrm{c}}=1 / 2\right)$ optimized COP is,

$$
\epsilon_{\widetilde{\chi}_{\max }}^{s y m}=\sqrt{1+\epsilon_{C}}-1 \equiv \epsilon_{C A},
$$

a result which could be considered as the counterpart for refrigerators of the CA efficiency $\eta=1-\sqrt{\tau}=1-$ $\sqrt{1-\eta_{C}}[19]$.

\section{B. CL refrigerator model without heat-leak (endoreversible model).}

Consider a reversible Carnot RE operating between the absolute temperatures $T_{\mathrm{hw}}$ and $T_{\mathrm{cw}}$ (see Fig. 1b) with $Q_{\mathrm{h}}$ 
and $Q_{\mathrm{c}}$ given by the heat transfer laws

$$
\begin{aligned}
& Q_{\mathrm{h}}=\sigma_{\mathrm{h}}\left(T_{\mathrm{hw}}^{k}-T_{\mathrm{h}}^{k}\right) t_{\mathrm{h}}=T_{\mathrm{h}}^{k} \sigma_{\mathrm{h}}\left(a_{\mathrm{h}}^{-k}-1\right) t_{\mathrm{h}} \geq 0, \\
& Q_{\mathrm{c}}=\sigma_{\mathrm{c}}\left(T_{\mathrm{c}}^{k}-T_{\mathrm{cw}}^{k}\right) t_{\mathrm{c}}=T_{\mathrm{c}}^{k} \sigma_{\mathrm{c}}\left(1-a_{\mathrm{c}}^{k}\right) t_{\mathrm{c}} \geq 0 .
\end{aligned}
$$

where the exponent $k \neq 0$ is a real number, $\sigma_{\mathrm{h}}$ and $\sigma_{\mathrm{c}}$ are the conductances in each process and $t_{\mathrm{h}}$ and $t_{\mathrm{c}}$ are the times at which isothermal processes are completed. Adiabatic processes' times are neglected. According to Eq. (3) $\chi$ is a function depending on the variables $a_{\mathrm{c}}$, $a_{\mathrm{h}}, t_{c} / t_{h}, k, \tau$ and $\sigma_{\mathrm{hc}}$. The endoreversible hypothesis $\Delta S_{T_{\mathrm{cw}}}=-\Delta S_{T_{\mathrm{hw}}}$ gives a constraint upon the time ratio $t_{\mathrm{c}} / t_{\mathrm{h}}:$

$$
\frac{t_{\mathrm{c}}}{t_{\mathrm{h}}}=\sigma_{\mathrm{hc}} a_{\mathrm{c}} a_{\mathrm{h}} \tau^{1-k}\left(\frac{a_{\mathrm{h}}^{-k}-1}{1-a_{\mathrm{c}}^{k}}\right),
$$

where $\sigma_{\mathrm{hc}} \equiv \sigma_{\mathrm{h}} / \sigma_{\mathrm{c}}$. Whenever a heat-leak is not present $T_{\mathrm{cw}} / T_{\mathrm{hw}}=a_{\mathrm{c}} a_{\mathrm{h}} \tau=\epsilon /(1+\epsilon)$ and the dependence on $a_{\mathrm{h}}$ can be replaced by $\epsilon$. In terms of $\alpha=\left(1+t_{\mathrm{h}} / t_{\mathrm{c}}\right)^{-1}$, Eq. (30) can be written as

$$
\frac{\alpha}{1-\alpha}=\sigma_{\mathrm{hc}} \tau^{k}\left(\frac{1+\epsilon}{\epsilon}\right)\left(\frac{\left(\frac{\epsilon \tau a_{\mathrm{c}}}{1+\epsilon}\right)^{k}-1}{1-a_{\mathrm{c}}^{k}}\right) .
$$

The optimization of $\chi=\chi\left(a_{\mathrm{c}}, \epsilon ; \sigma_{\mathrm{hc}}, \tau, T_{\mathrm{h}}, k\right)$ is achieved through $a_{\mathrm{c}}$ and $\epsilon$ by solving $\left(\frac{\partial \chi}{\partial a_{\mathrm{c}}}\right)_{\epsilon}=0$ for $a_{\mathrm{c}}$ and $\left(\frac{\partial \chi}{\partial \epsilon}\right)_{a_{\mathrm{c}}}=0$ for $\epsilon$. From the first condition $\left(a_{\mathrm{c}}^{*}\right)$ we obtain $\chi^{*}$ as a function of $\epsilon$

$$
\begin{gathered}
\chi^{*}\left(\epsilon ; \sigma_{\mathrm{hc}}, \tau, T_{\mathrm{h}}, k\right)=\chi\left(a_{\mathrm{c}}^{*}, \epsilon ; \sigma_{\mathrm{hc}}, \tau, T_{\mathrm{h}}, k\right) \\
=\sigma_{\mathrm{h}} T_{\mathrm{h}}^{k} \epsilon \frac{\tau^{k}-\left(\frac{\epsilon}{1+\epsilon}\right)^{k}}{\left(\sqrt{\sigma_{\mathrm{hc}}}+\left(\frac{\epsilon}{1+\epsilon}\right)^{\frac{k-1}{2}}\right)^{2}},
\end{gathered}
$$

which shows a unique maximum, obtained by solving numerically $\left(\frac{\partial \chi^{*}}{\partial \epsilon}\right)_{a_{c}^{*}}=0$ for $\epsilon$. Only for the cases $\sigma_{\mathrm{hc}} \rightarrow\{0, \infty\}$ the solutions are analytical, which are displayed in Fig. 3a (dot-dashed curves, green online). In Fig. 3, for $\sigma_{\text {hc }} \rightarrow \infty$ beyond $k \approx 2$ there are no mathematical solutions for $\chi_{\max }$, meanwhile, in the case $\sigma_{\mathrm{hc}} \rightarrow 0$ below $k=-1$ the solutions are non-physical $(\epsilon<0)$. For any $\sigma_{\mathrm{hc}} \in(0, \infty)$ all $\epsilon_{\chi_{\max }}$ 's are located between those curves. It can be seen that the LD bounds provided by the LD-model (Eqs. (26) and (27)) are fulfilled by the endoreversible RE only for $k=-1$ which also occurred for HE's. Outside the region bounded by the two curves no $\sigma_{\text {hc }}$ value can reproduce the LD-model COP's. The Newtonian heat transfer law $(k=1)$ is the only case where all possible values of $\sigma_{\mathrm{hc}}$ give the same $\mathrm{COP}\left(\epsilon_{C A}\right)$. On the other hand, in the LD model $\epsilon_{C A}$ is attached only to $\widetilde{\Sigma}_{\mathrm{c}}=1 / 2$. To reconcile these situations it will be shown that there is only one $\sigma_{\mathrm{hc}}$ corresponding to a symmetric dissipation.
From the LD optimization $\alpha$ and $\widetilde{t}$ for the case $\widetilde{\Sigma}_{\mathrm{c}}=$ $1 / 2$ are

$$
\begin{aligned}
\alpha_{\widetilde{\chi}_{\text {max }}}^{\text {sym }} & =\frac{1+\sqrt{1-\tau}}{2+\sqrt{1-\tau}}, \\
\widetilde{t}_{\widetilde{\chi}_{\text {max }}^{\text {sym }}} & =\frac{2+\sqrt{1-\tau}}{\sqrt{1-\tau}} .
\end{aligned}
$$

then, $a_{\mathrm{c}}$ can be computed according to Eq. (16) and $a_{\mathrm{h}}$ is obtained from the condition $T_{\mathrm{cw}} / T_{\mathrm{hw}}=a_{\mathrm{c}} a_{\mathrm{h}} \tau=\frac{\epsilon}{1+\epsilon}=$ $1-\sqrt{1-\tau}$ by using $\epsilon=\epsilon_{C A}$, then,

$$
\begin{aligned}
& a_{\mathrm{c}, \chi_{\max }}^{\text {sym }}=\frac{2+\sqrt{1-\tau}}{2(1+\sqrt{1-\tau})} \\
& a_{\mathrm{h}, \chi_{\max }}^{\text {sym }}=\frac{2}{2+\sqrt{1-\tau}}
\end{aligned}
$$

that is, the above $a_{\mathrm{c}}$ and $a_{\mathrm{h}}$ are obtained from the LD model optimization and the endoreversible hypothesis. If the optimization of both models were equivalent it is expected that by means of Eq. (31) the corresponding $\sigma_{\mathrm{hc}}$, given by the following expression

$$
\sigma_{\mathrm{hc}}^{\text {sym }}=\tau^{k}\left(\frac{1+\sqrt{1-\tau}}{1-\sqrt{1-\tau}}\right)\left(\frac{2^{k}-\left(\frac{2+\sqrt{1-\tau}}{1+\sqrt{1-\tau}}\right)^{k}}{(2+\sqrt{1-\tau})^{k}-2^{k}}\right),
$$

would reproduce $\epsilon_{C A}$. In Fig. $3 \mathrm{~b}$ it can be seen that the $\epsilon_{C A}$ value is not perfectly reproduced but only in the cases $k=\{-1,1\}$. However, there is a good agreement in the region between these two values, a common feature with the HE case. For $k=1, \sigma_{\mathrm{hc}}=1+\sqrt{1-\tau}$ and for $k=-1, \sigma_{\mathrm{hc}}=(1-\sqrt{1-\tau})^{-2}$.

\section{CL refrigerator with heat-leak.}

Now, let us consider a heat-leak of the same kind than $Q_{\mathrm{c}}$ and $Q_{\mathrm{h}}$,

$$
\begin{aligned}
Q_{\mathrm{L}} & =\sigma_{\mathrm{L}}\left(T_{\mathrm{h}}^{k}-T_{\mathrm{c}}^{k}\right)\left(t_{\mathrm{h}}+t_{\mathrm{c}}\right) \\
& =T_{\mathrm{h}}^{k} \sigma_{\mathrm{L}}\left(1-\tau^{k}\right)\left(t_{\mathrm{h}}+t_{\mathrm{c}}\right) \geq 0,
\end{aligned}
$$

where $\sigma_{\mathrm{L}}$ is the heat-leak conductance. Then

$$
\begin{aligned}
\widetilde{Q}_{\mathrm{L}}=\frac{Q_{\mathrm{L}}}{Q_{\mathrm{c}}} & =a_{\mathrm{c}} \frac{T_{\mathrm{h}}^{k} \sigma_{\mathrm{L}}\left(1-\tau^{k}\right)\left(t_{\mathrm{h}}+t_{\mathrm{c}}\right)}{T_{\mathrm{c}}^{k} \sigma_{\mathrm{c}}\left(a_{\mathrm{c}}^{k}-1\right) t_{\mathrm{c}}} \\
& =\frac{a_{\mathrm{c}} \sigma_{\mathrm{Lc}}\left(1-\tau^{k}\right)}{\tau^{k}\left(a_{\mathrm{c}}^{k}-1\right)}\left(\frac{t_{\mathrm{h}}}{t_{\mathrm{c}}}+1\right)
\end{aligned}
$$

where $\sigma_{\mathrm{Lc}} \equiv \sigma_{\mathrm{L}} / \sigma_{\mathrm{L}}$, in this case $R$ takes into account the heat transferred by $Q_{L}$, thus, $\epsilon$ and $\chi$ read as

$$
\begin{gathered}
\epsilon \equiv \frac{R}{P_{i n}}=\frac{\left(Q_{\mathrm{c}}-Q_{\mathrm{L}}\right)}{\left(Q_{\mathrm{h}}-Q_{\mathrm{c}}\right)}=\frac{\left(1-\widetilde{Q}_{\mathrm{L}}\right)}{\left(\frac{Q_{\mathrm{h}}}{Q_{\mathrm{c}}}-1\right)}, \\
\chi \equiv \epsilon R=\frac{R^{2}}{P_{i n}}=\frac{\left(1-\widetilde{Q}_{\mathrm{L}}\right)^{2} Q_{\mathrm{c}}}{\left(\frac{Q_{\mathrm{h}}}{Q_{\mathrm{c}}}-1\right) t},
\end{gathered}
$$




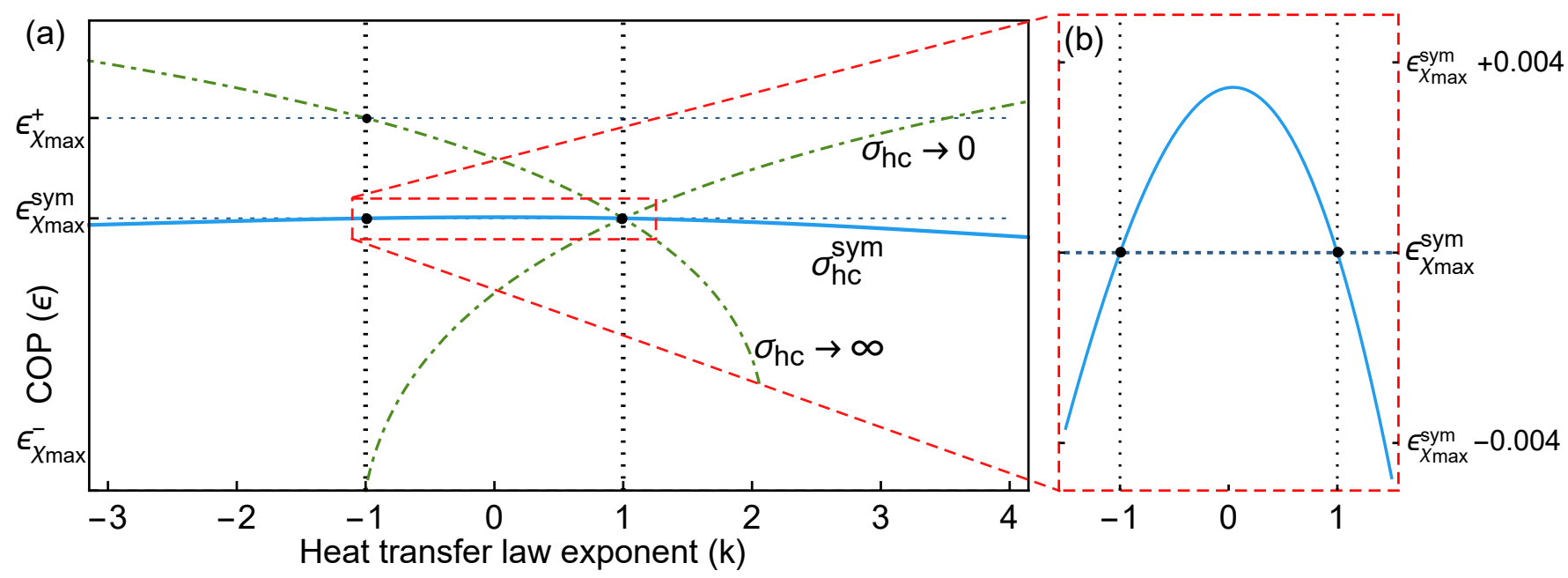

FIG. 3. In a) the upper and lower bounds of the COP for the CL RE at maximum- $\chi$ regime versus the heat transfer law exponent $k$. The $\sigma_{\mathrm{hc}} \rightarrow\{0, \infty\}$ along with the $\sigma_{\mathrm{hc}}^{\text {sym }}$ case (Eq. (37)) are plotted. The bounds provided by Eqs. (26) and (27) are labeled. In b) a close view of $\epsilon_{\chi \max }$ using $\sigma_{\mathrm{hc}}^{s y m}$ from Eq. (37). In all cases the representative value of $\tau=0.7$ is used.

where $R=\left(Q_{\mathrm{c}}-Q_{\mathrm{L}}\right) / t$. The heat-leak diminishes the $\mathrm{COP}$ and is responsible for the loop-like COP versus $\chi$ curves.

The maximization of Eq. (41) is obtained through $a_{\mathrm{h}}$ and $a_{\mathrm{c}}$ by solving numerically $\left(\frac{\partial \chi}{\partial a_{\mathrm{c}}}\right)_{a_{\mathrm{h}}}=0$ for $a_{\mathrm{c}}$ and $\left(\frac{\partial \chi}{\partial a_{\mathrm{h}}}\right)_{a_{\mathrm{c}}}=0$ for $a_{\mathrm{h}}$. In the limit situations $\left\{\sigma_{\mathrm{c}}, \sigma_{\mathrm{h}}\right\} \rightarrow$ $\infty$ the solutions can be obtained analytically but in the general case their obtaining requires numerical methods. In Fig. 4 they are depicted for three cases. The effect of the heat-leak is more noticeable for higher COP values, meanwhile for lower values it is scarce.

The similarity between the above results for RE and those reported for HE [44] strengthen the road toward unified studies of heat devices. In this sense, the $\Omega$ function will provide useful insights due to the capability of obtaining analytical closed expressions.

\section{MAXIMUM- $\Omega$ REGIME}

From Eq. (6) and since $P_{i n}=W / t=\left(Q_{\mathrm{h}}-Q_{\mathrm{c}}\right) / t$, $R=Q_{\mathrm{c}} / t$ and $\epsilon=Q_{\mathrm{c}} / W=R / P_{\text {in }}$, if $\epsilon_{\max }=\epsilon_{C}$, then

$$
\Omega^{R E}=\Omega=2 R-\frac{\tau}{1-\tau} P_{i n} .
$$

\section{A. Maximum- $\Omega$ regime for a $L D R E$}

From Eqs. (21) and (22) $\widetilde{\Omega} \equiv \Omega t /\left(T_{\mathrm{c}} \Delta S \widetilde{t}\right)$ is given by

$$
\widetilde{\Omega}=\frac{\tau}{\widetilde{t}}\left[1-\frac{\widetilde{\Sigma}_{\mathrm{c}}}{\alpha \widetilde{t}}\left(\frac{2-\tau}{1-\tau}\right)-\frac{1-\Sigma_{\mathrm{c}}}{(1-\alpha)(1-\tau) \widetilde{t}}\right] .
$$

Its optimization is achieved through $\alpha$ and $\tilde{t}$ as in the $\widetilde{\chi}_{\max }$ case by solving simultaneously the conditions $\left(\frac{\partial \widetilde{\Omega}}{\partial \alpha}\right)_{\widetilde{t}}=0$ and $\left(\frac{\partial \widetilde{\Omega}}{\partial \widetilde{t}}\right)_{\alpha}=0$, leading to

$$
\alpha_{\widetilde{\Omega}_{\max }}=\frac{1}{1+\sqrt{\frac{1-\widetilde{\Sigma}_{\mathrm{c}}}{\widetilde{\Sigma}_{\mathrm{c}}(2-\tau)}}}
$$

$$
\widetilde{t}_{\widetilde{\Omega}_{\text {max }}}=\frac{2}{1-\tau}\left(\sqrt{1-\widetilde{\Sigma}_{\mathrm{c}}}+\sqrt{\widetilde{\Sigma}_{\mathrm{c}}(2-\tau)}\right)^{2}
$$

$\widetilde{\Omega}_{\max }$ and $\epsilon_{\widetilde{\Omega}_{\max }}$ are functions of $\widetilde{\Sigma}_{\mathrm{c}}$ and $\tau$, the latter being an increasing function of $\widetilde{\Sigma}_{\mathrm{c}}$ bounded by

$$
\epsilon_{\widetilde{\Omega}_{\max }}^{-}=\frac{2}{3} \epsilon_{C} \leq \epsilon_{\widetilde{\Omega}_{\max }} \leq \frac{3+2 \epsilon_{C}}{4+3 \epsilon_{C}} \epsilon_{C}=\epsilon_{\widetilde{\Omega}_{\max }}^{+},
$$

where the lower and upper bounds are obtained in the limits $\widetilde{\Sigma}_{\mathrm{c}} \rightarrow 0$ and $\widetilde{\Sigma}_{\mathrm{c}} \rightarrow 1$, respectively. In the symmetric case $\widetilde{\Sigma}_{\mathrm{c}}=1 / 2$,

$$
\epsilon_{\widetilde{\Omega}_{\text {max }}}^{\text {sym }}=\frac{\epsilon_{C}}{\sqrt{\left(1+\epsilon_{C}\right)\left(2+\epsilon_{C}\right)}-\epsilon_{C}} .
$$

These limits are in agreement with those reported in [53], but obtained in a quite different manner.

\section{B. Maximum- $\Omega$ regime for a CL endoreversible $\mathrm{RE}$}

The $\Omega$ function is given by

$$
\Omega=2 R-\frac{\tau}{1-\tau} P_{i n}=\frac{Q_{\mathrm{c}}}{(1-\tau) t}\left(2-\tau-\frac{\tau Q_{\mathrm{h}}}{Q_{\mathrm{c}}}\right) .
$$




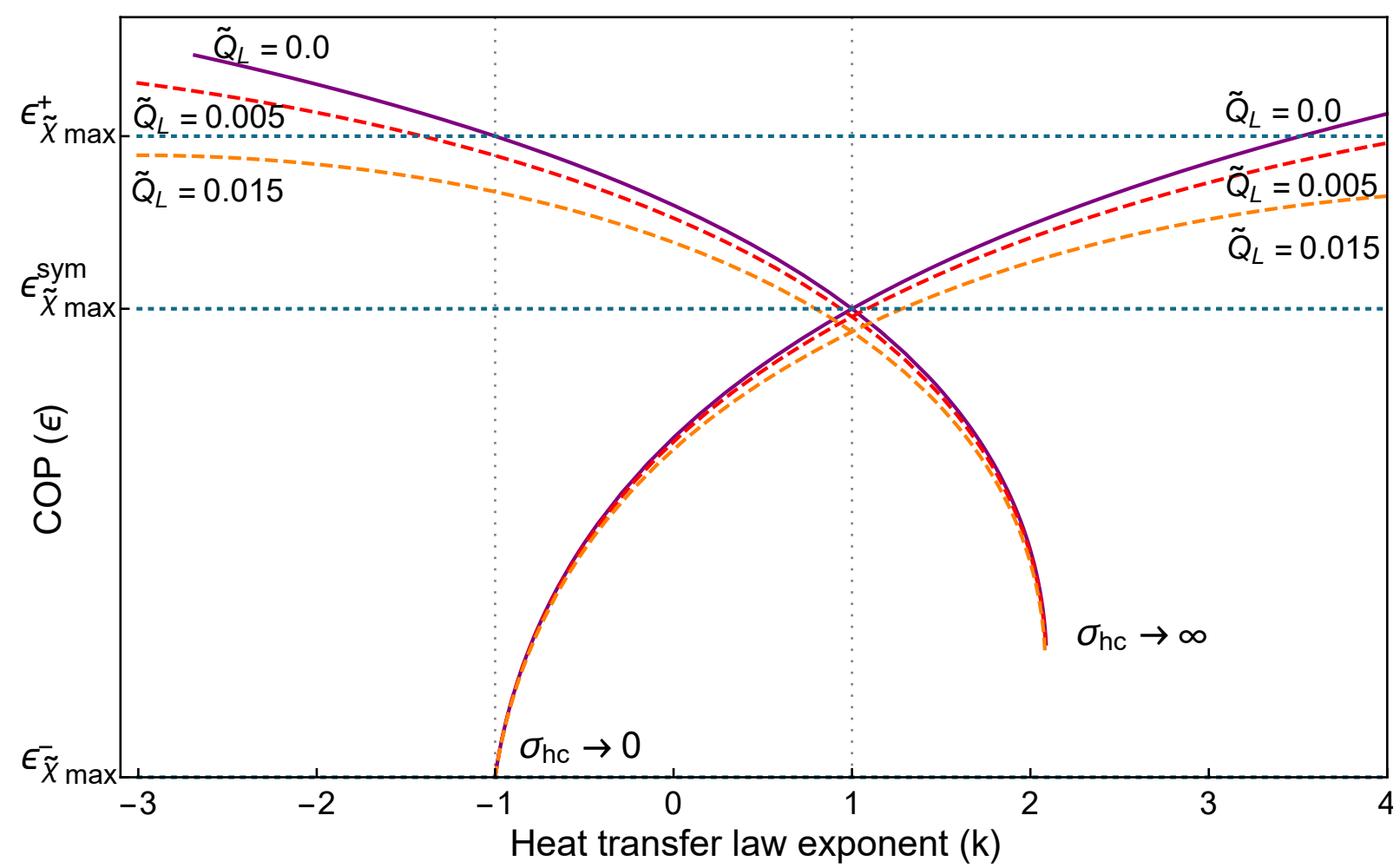

FIG. 4. Influence of the heat-leak on the upper and lower bounds of the COP at the $\chi_{\max }$ regime. The cases $\sigma_{\mathrm{L}}=$ $\{0,0.005,0.015\}$ are displayed. The representative value $\tau=0.7$ is used.

From the endoreversible hypothesis $\frac{Q_{\mathrm{c}}}{Q_{\mathrm{h}}}=\frac{T_{\mathrm{cw}}}{T_{\mathrm{hw}}}=a_{\mathrm{c}} a_{\mathrm{h}} \tau$ and since $\epsilon=\left(\frac{T_{\mathrm{hw}}}{T_{\mathrm{cw}}}-1\right)^{-1}$ it follows that

$$
\frac{Q_{\mathrm{h}}}{Q_{\mathrm{c}}}=\frac{T_{\mathrm{hw}}}{T_{\mathrm{cw}}}=\frac{1+\epsilon}{\epsilon},
$$

and by means of Eqs. (28) and (29) it leads to

$$
\frac{t_{\mathrm{h}}}{t_{\mathrm{c}}}=\frac{\tau^{k} \phi(1-x)}{\sigma_{\mathrm{hc}}\left(x \tau^{k} \phi^{k}-1\right)}
$$

where $\phi \equiv \frac{1+\epsilon}{\epsilon}$ and $x \equiv a_{\mathrm{c}}^{k}$. Then, $\Omega$ is given by

$$
\Omega=\frac{T_{\mathrm{c}}^{k} \sigma_{h} \tau^{k}}{1-\tau}\left(\frac{(1-x)(2-\tau-\tau \phi)}{1+\frac{\tau^{k} \phi(1-x)}{\sigma_{\mathrm{hc}}\left(x \tau^{k} \phi^{k}-1\right)}}\right) .
$$

Its maximization is achieved by solving $\left(\frac{\partial \Omega}{\partial x}\right)_{\phi}=0$ for $x$ and $\left(\frac{\partial \Omega}{\partial \phi}\right)_{x}=0$ for $\phi$. From the first condition one obtains $x^{*}$, given by

$$
\begin{aligned}
x^{*} & =\frac{\sqrt{\phi^{1-k}}+\tau^{-k} \phi^{-k} \sqrt{\sigma_{\mathrm{hc}}}}{\sqrt{\phi^{1-k}}+\sqrt{\sigma_{\mathrm{hc}}}}, & & k>0, \\
& =\frac{\sqrt{\phi^{1-k}}-\tau^{-k} \phi^{-k} \sqrt{\sigma_{\mathrm{hc}}}}{\sqrt{\phi^{1-k}}-\sqrt{\sigma_{\mathrm{hc}}}}, & & k<0,
\end{aligned}
$$

both cases lead to the same $\Omega^{*}$ function, given by

$$
\begin{array}{r}
\Omega^{*}\left(\phi ; T_{\mathrm{h}}, \sigma_{\mathrm{c}}, \sigma_{\mathrm{hc}}, \tau, k\right)= \\
\frac{T_{\mathrm{h}}^{k} \sigma_{\mathrm{c}} \sigma_{\mathrm{hc}}}{1-\tau}\left(\frac{(2-\tau-\tau \phi)\left(\tau^{k}-\phi^{-k}\right)}{\left(\sqrt{\phi^{1-k}}-\sqrt{\sigma_{\mathrm{hc}}}\right)^{2}}\right) .
\end{array}
$$

Finally, $\epsilon_{\Omega_{\text {max }}}\left(\sigma_{\mathrm{hc}}, \tau, k\right)$ is obtained from the condition $\left(\frac{\partial \Omega^{*}}{\partial \phi}\right)_{x^{*}}=0$. In Fig. 5 the limiting cases $\sigma_{\mathrm{hc}} \rightarrow\{0, \infty\}$ of $\epsilon_{\Omega_{\max }}$ are depicted (continuous lines, purple online), these two curves bound any other possible values of the COP; for $k<-1$ the lower bound is given by $\epsilon=0$. Once more, the region of heat transfer laws where the endoreversible and the LD RE results overlap is limited. Only at $k=-1$ the upper and lower LD bounds are recovered.

\section{Maximum- $\Omega$ for a CL RE with heat-leak}

From Eq. (48), with the use of Eqs. (28), (29), (30) and (38),

$$
\begin{gathered}
\Omega=\frac{2\left(Q_{\mathrm{c}}-Q_{\mathrm{L}}\right)}{t}-\frac{\tau Q_{\mathrm{c}}}{(1-\tau) t}\left(\frac{Q_{\mathrm{h}}}{Q_{\mathrm{c}}}-1\right)= \\
\sigma_{\mathrm{c}} T_{\mathrm{c}}^{k}\left[\sigma_{\mathrm{hc}} \frac{(2-\tau) a_{\mathrm{c}} a_{\mathrm{h}}-1}{\left(1-\tau^{k}\right)\left(\frac{\sigma_{\mathrm{hc}} a_{\mathrm{h}} a_{\mathrm{h}}}{1-a_{\mathrm{c}}^{k}}+\frac{\tau^{k-1}}{a_{\mathrm{h}}^{-k}-1}\right)}-2 \sigma_{\mathrm{Lc}} \frac{1-\tau^{k}}{\tau^{k}}\right]
\end{gathered}
$$




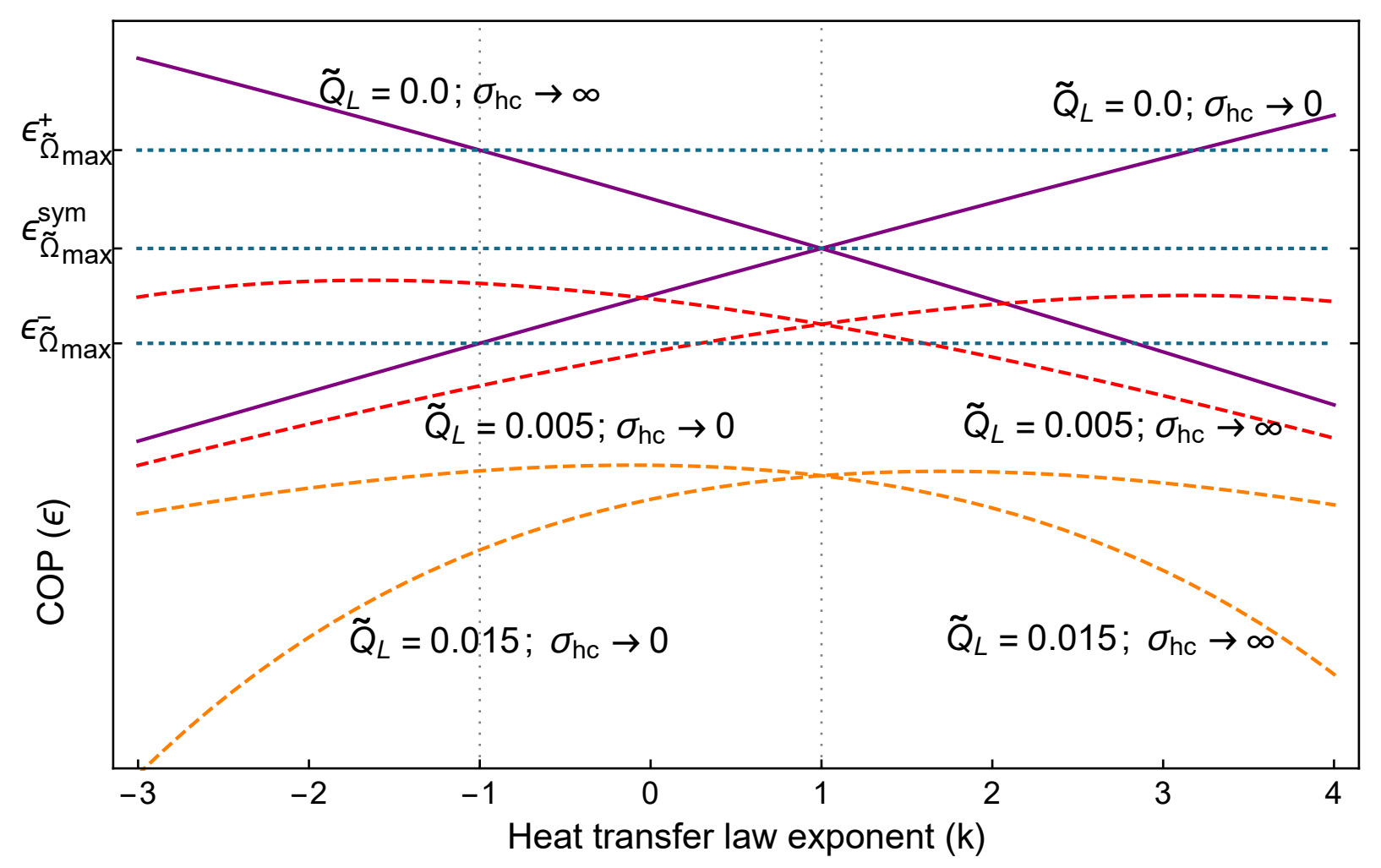

FIG. 5. COP of the endoreversible $\left(Q_{\mathrm{L}}=0\right)$ RE operating at $\Omega_{\max }$ and two cases with $Q_{\mathrm{L}}=\{0.005,0.015\}$. The representative value of $\tau=0.7$ is used.

that is, the heat-leak contributes with an additive term which does not alter the optimization in terms of $a_{\mathrm{c}}$ and $a_{\mathrm{h}}$. In Fig. 5 it is depicted the influence of the heat-leak on $\epsilon$ for $\sigma_{\mathrm{hc}} \rightarrow\{0, \infty\}$. A noticeable difference with respect to the $\chi_{\max }$ (Fig. 4 ) is that here the entire bounds are lowered by the heat-leak.

Below, we present a comparison between the two regimes focused on the role of dissipation symmetries and the entropy generation within the LD-model framework.

\section{VI. $\chi_{\max }$ VERSUS $\Omega_{\max }$ FOR A LD RE.}

A comparison between energetic properties involving entropy production and COP in the two regimes in the $\mathrm{LD}$ model is quite illustrative to get insights about the influence of the dissipation symmetries beyond the particularities on the heat transfer mechanisms, taking advantage that the LD approximation is good for certain set of them.

In Fig. 6(a-f) we show the influence of the dissipation symmetries on $\alpha, \widetilde{t}, \widetilde{P}_{i n}, \widetilde{R}, \epsilon$ and $\dot{\overrightarrow{\Delta S}}$ under the $\widetilde{\chi}_{\max }$ and $\widetilde{\Omega}_{\max }$ regimes (dashed lines and continuous lines, respectively).

As expected, the operation time and COP are larger for the latter. When the dissipation is mostly located in the contact with the cold reservoir $\left(\widetilde{\Sigma}_{\mathrm{c}} \rightarrow 1\right)$ the entropy generation is the lesser (Fig. 6f), but not necessarily at- tached to the smallest cooling power or power-input (see Fig. 6(c-d)) nor the largest total-time $\tilde{t}$ (Fig. 6b). Notice that $\epsilon$ increases and the entropy production diminishes as the contact-time $\alpha$ increases.

On the right side of Fig. 6 the comparison of both regimes is complemented. Fig. $6(\mathrm{~g}-\mathrm{h})$ offers a measure of the distance that each time variable has to be shifted in order to change the operation regime. Note the existence of well defined maxima, while the ratios depicted in Fig. 6( $i-l)$ are monotonous functions bounded by the limiting asymmetrical dissipation cases $\widetilde{\Sigma}_{\mathrm{c}}=\{0,1\}$. Depending on the dissipation symmetry the transition of one regime to another can offer certain advantages. For example, for small values of $\widetilde{\Sigma}_{\mathrm{c}}$ (when the major part of the dissipation occur at the hot reservoir) the transition gives the largest gain in terms of the COP, however, it requires also the largest changes in the cooling power, power input and entropy production. Nevertheless the changes of $\alpha$ as well as the total operation times are small and a passing from one regime into the other demands small changes of these control parameters. In the opposite situation, when $\widetilde{\Sigma}_{\mathrm{c}} \rightarrow 1$ the switching of regime demands also small variations of $\alpha$ and $\widetilde{t}$, with a small gaining in the COP, but also with small increments of the entropy production, cooling power and power input, resulting also in a suitable case since in each regime the COP is the largest (see Fig. 6e). 

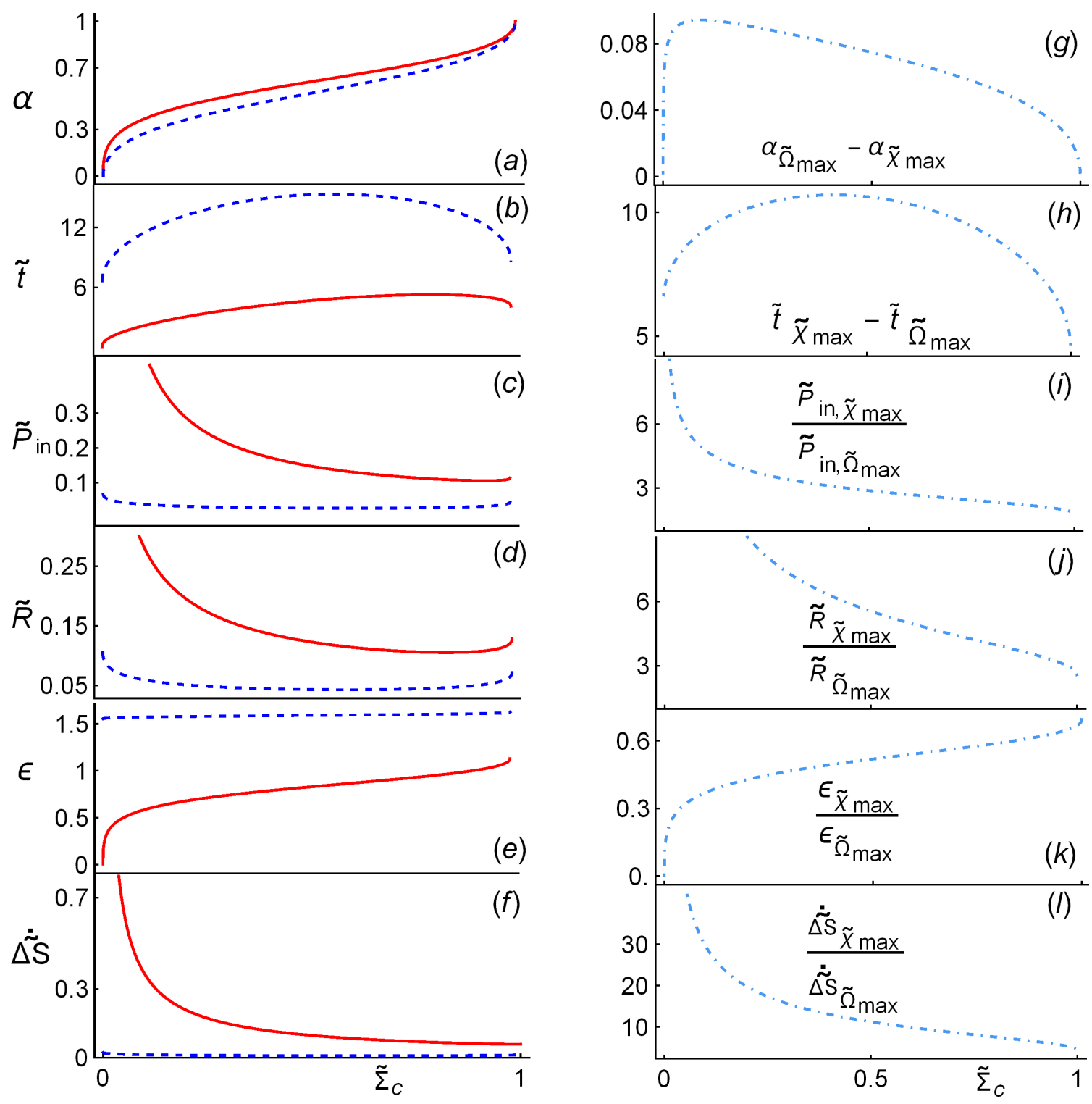

FIG. 6. Left: $\widetilde{\Omega}_{\max }$ (dashed lines, blue online) and $\widetilde{\chi}_{\max }$ (continuous lines, red online). Right: Comparison between $\widetilde{\Omega}_{\max }$ and $\tilde{\chi}_{\max }$. In all cases we use $\tau=0.7$.

By incorporating the study of the entropy production, relevant information regarding the benefits of having certain dissipation symmetries arise. In Fig. 7 a 3D-plot of the total entropy production is shown under the timeconstraints $\widetilde{t}=\widetilde{t}_{\widetilde{\chi}_{\max }}$ and $\widetilde{t}=\widetilde{t}_{\widetilde{\Omega}_{\max }}$. Over the surfaces, curves of constant COP are displayed. As can be seen, the entropy can significantly increase or decrease despite of having the same COP. This is more noticeable for the $\chi_{\max }$ regime. The curve $\epsilon=0$ bounds the physical region of interest. As the COP value increases the set of possible $\alpha-\widetilde{\Sigma}_{\mathrm{c}}$ combinations that produce such COP bound a narrower region, until the limiting situation where only $\alpha \rightarrow \widetilde{\Sigma}_{\mathrm{c}} \rightarrow 1$ produce the upper bounds $\epsilon_{\widetilde{\Omega}_{\max }, \widetilde{\chi}_{\max }}$. As can be seen, the maximum achievable COP does not correspond to the minimum entropy production.

\section{CONCLUDING REMARKS}

A relation between the variables that describe the lowdissipation RE model and those that describe the Carnotlike $\mathrm{RE}$ has been presented from considerations on the entropy generation. The physical space of the variables is consistent for both models. However, from the optimization process the correspondence between both set of variables is only valid for the heat transfer laws with exponent $k=\{-1,1\}$ and very close in the region $(-1,1)$. Outside this range of exponents there are COP values stemming from the Carnot-like RE that cannot be recovered from the low-dissipation model. These results are consistent with previous studies on HE's, reinforcing the concept of the unified criteria of merit by means of the $\chi$ 


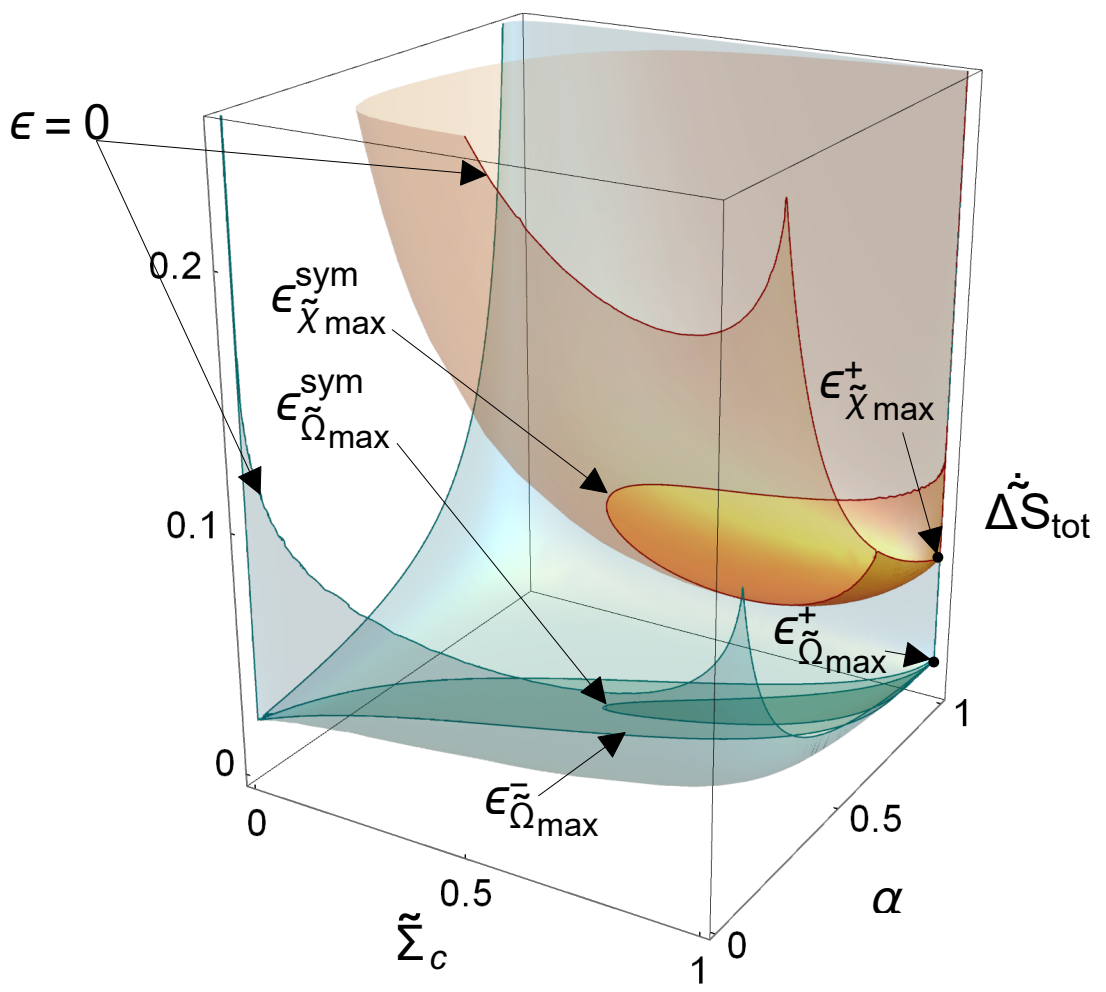

FIG. 7. $\dot{\overrightarrow{\Delta S}}_{t o t}$ under the time-constraints $\widetilde{t}=\widetilde{t}_{\widetilde{\chi}_{\max }}$ (upper surface) and $\widetilde{t}=\widetilde{t}_{\widetilde{\Omega}_{\max }}$ (lower surface). Over each surface the level curves denote the configurations of $\widetilde{\Sigma}_{\mathrm{c}}$ and $\alpha$ that produce the same COP. We use the representative value $\tau=0.7$.

and the $\Omega$ functions.

The role of the entropy generation has been addressed together with the criteria of merit, allowing for a complementary vision of the role of dissipation symmetries and the nature of the irreversibilities in the energetics of these kind of energy converters. In this way a global study of dissipation symmetries' effects on the COP, entropy generation, power input and cooling power can be obtained. As a result, upper and lower bounds of the relative gaining or loosing of such quantities in a change of operation regime can be evaluated.

\section{ACKNOWLEDGEMENT}

J. G.-A. acknowledge CONACYT-MÉXICO for partial financial support. We acknowledge financial support from Junta de Castilla y Leon under project SA017P17.

\section{REFERENCES}

[1] L. A. Correa, J. P. Palao, D. Alonso, G. Adesso, Quantum-enhanced absorption refrigerators, Scientific Reports 4 (2014) 3949, DOI:10.1038/srep03949

[2] L. A. Correa, J. P. Palao, D. Alonso, Internal dissipation and heat leaks in quantum thermodynamic cycles, Phys Rev E 92(3) (2015) 032136, DOI:10.1103/PhysRevE.92.032136

[3] P. Liuzzo-Scorpo, L. A. Correa, R. Schmidt, G. Adesso, Thermodynamics of quantum feedback cooling Entropy 18(2) (2016) 48, DOI:10.3390/e18020048

[4] A. E. Allahverdyan, K. Hovhannisyan, G. Mahler, Optimal refrigerator, Phys Rev E 81 (2010) 051129, DOI:10.1103/PhysRevE.81.051129
[5] R. Long, W. Liu, Performance of quantum Otto refrigerators with squeezing, Phys Rev E 91 (2015) 062137, DOI:10.1103/PhysRevE.91.062137

[6] R. Long, W. Liu, Performance of micro two-level heat devices with prior information Phys Lett A 379 (2015) 1979-1982, DOI:10.1016/j.physleta.2015.06.012

[7] O. Abah, E. Lutz, Energy ecient quantum machines EPL 118 (2017) 40005, DOI:10.1209/0295-5075/118/40005

[8] E. Torrontegui, R. Kosloff, Quest for absolute zero in the presence of external noise, Physical Review E $8 \mathbf{8}$ (2013) 032103, DOI:10.1103/PhysRevE.88.032103

[9] R. Kosloff, A. Levy, Quantum Heat Engines and Refrigerators: Continuous Devices, Annual Review of Physical 
Chemistry 65(1) (2014) 365-393, DOI:10.1146/annurevphyschem-040513-103724

[10] G. Su, T. Liao, L. Chen, J. Chen, Performance evaluation and optimum design of a new-type electronic cooling device, Energy 101 (2016) 421-426, DOI:doi:10.1016/j.energy.2016.02.059

[11] S. Su, Y. Zhang, J. Chen, T. M. Shih, Thermal electrontunneling devices as coolers and amplifiers Scientific Reports 6 (2016) 21425, DOI:10.1038/srep21425

[12] J. M. Gordon, K. C. Ng, Cool Thermodynamics Cambridge International Science Publishing, Cambridge, UK, 2001, ISBN 9781898326908

[13] B. Sahin, A. Kodal, Thermoeconomic optimization of a two stage combined refrigeration system: a finitetime approachInternational Journal of Refrigeration $\mathbf{2 5}$ (2002) 872-877, DOI:10.1016/S0140-7007(01)00062-7

[14] P. K. Bhardwaj, S. C. Kaushik, S. Jain, Finite time optimization of an endoreversible and irreversible vapour absorption refrigeration system, Energy Conversion and Management 44(7) (2003) 1131-1144, DOI:10.1016/S0196-8904(02)00101-2

[15] C. Petre, M. Feidt, M. Costea, S. Petrescu, A model for study and optimization of real-operating refrigeration machines, International Journal of Energy Research 33 (2) (2009) 173-179, DOI:10.1002/er.1433

[16] Y. Ust, Performance analysis and optimization of irreversible air refrigeration cycles based on ecological coefficient of performance criterion, $A p$ plied Thermal Engineering 29 (2009) 47-55, DOI:10.1016/j.applthermaleng.2008.01.024

[17] S. Sheng, Z. C. Tu, Universality of energy conversion efficiency for optimal tight-coupling heat engines and refrigerators, J Phys A: Mathematical and Theoretical 46(40) (2013) 402001, DOI:10.1088/1751-8113/46/40/402001

[18] Lingen Chen, Dongliang Ni, Zelong Zhang, and Fengrui Sun 2016 Exergetic performance optimization for new combined intercooled regenerative Brayton and inverse Brayton cycles Applied Thermal Engineering 102, 447453, DOI:10.1016/j.applthermaleng.2016.03.058

[19] Y. Izumida, K. Okuda, J. M. M. Roco, A. Calvo Hernández, Heat devices in nonlinear irreversible thermodynamics, Phys. Rev. E 91 (2015) 052140, DOI:10.1103/PhysRevE.91.052140

[20] L. Chen, Z. Ding, F. Sun, A generalized model of an irreversible thermal Brownian refrigerator and its performance, Applied Mathematical Modelling 35 (2011) 29452958, DOI:10.1016/j.apm.2010.12.008

[21] S. Rana, P. S. Pal, A. Saha, A. M. Jayannavar, Anomalous Brownian Refrigerator Physica A 444 (2015) 783798, DOI:10.1016/j.physa.2015.10.095

[22] R. Long, B. Li, W. Liu, Performance analysis for Feynman's ratchet as a refrigerator with heat leak under different figure of merits, Applied Mathematical Modelling 40 (2016) 10437-10446, DOI:10.1016/j.apm.2016.07.027

[23] E. Açıkkalp, A. F. Savaş, N. Caner, H. Yamık, Assessment of nano-scale Stirling refrigerator using working fluid as Maxwell-Boltzmann gases by thermo-ecological and sustainability criteria, Chemical Physics Letters $\mathbf{6 5 8}$ (2016) 303-308, DOI:10.1016/j.cplett.2016.06.064

[24] A. Dalkiran, E. Açıkkalp, A. F. Savaş, Analysis of a nano-scale thermo-acoustic refrigerator, Int. J. Refrig. 66 (2016) 1-9, DOI:10.1016/j.ijrefrig.2016.01.022

[25] M. H. Ahmadi, M. A. Ahmadi, A. Malekia, F. Pourfayaz, M. Bidi, E. Açıkkalp, Exergetic sustainability evaluation and multi-objective optimization of performance of an irreversible nanoscale Stirling refrigeration cycle operating with Maxwell-Boltzmann gas, Renewable and Sustainable Energy Reviews 78 (2017) 80-92, DOI:doi:10.1016/j.rser.2017.04.097

[26] Z. Xu, J. Guo, G. Lin, J. Chen, Optimal thermoeconomic performance of an irreversible regenerative ferromagnetic Ericsson refrigeration cycle Journal of Magnetism and Magnetic Materials 409 (2016) 71-79, DOI:10.1016/j.jmmm.2016.02.063

[27] H. Ouerdane , Y. Apertet, C. Goupil, A. Michot, A. Abbout, A linear nonequilibrium thermodynamics approach to optimization of thermoelectric device, Thermoelectric Nanomaterials, K. Koumoto et T. Mori Editors. Springer Series in Materials Science 182 (2103) 323-351, ISBN:978-3-642-37537-8

[28] Y. Apertet, H. Ouerdane, A. Michot, C. Goupil, P. Lecoeur, On the efficiency at maximum cooling power, EPL 103(4) (2103) 40001, DOI:10.1209/02955075/103/40001

[29] I. Iyyappan, M. Ponmurugan, Thermoelectric energy converters under a trade-off figure of merit with broken time-reversal symmetry, DOI:https://arxiv.org/abs/1604.07551 arXiv:1604.07551 (2016)

[30] U. Eicker, D. Pietruschka, A. Schmitt, M. Haag, Comparison of photovoltaic and solar thermal cooling systems for office buildings in different, Solar Energy 118 (2017) 243-255, DOI:10.1016/j.solener.2015.05.018

[31] F. M. Montagnino, Solar cooling technologies. Design, application and performance of existing projects, Solar Energy, (2017) In Press, DOI:10.1016/j.solener.2017.01.033

[32] L. Chen, C. Wu, F. Sun, Finite time thermodynamic optimization or entropy generation minimization of energy systems, Journal of Non-Equilibrium Thermodynamics 24 (1999) 327-359, DOI:10.1515/JNETDY.1999.020

[33] A. Durmayaz, O.S. Sogut, B. Sahin, H. Yavuz 2004 Optimization ofthermal systems based on finite-time thermodynamics and thermo-economics Progress in Energy and Combustion Science 30, 175-217 ; DOI:

[34] F. L. Curzon, B. Ahlborn, Efficiency of a Carnot engine at maximum power output Am J Phys 43 (1975) 22, DOI:10.1119/1.10023

[35] M. Esposito, R. Kawai, K. Lindenberg, C. Van den Broeck 2010 Efficiency at Maximum Power of LowDissipation Carnot Engines Phys. Rev. Lett. 105 150603, DOI:10.1103/PhysRevLett.105.150603

[36] C. de Tomás, A. Calvo Hernández, J. M. M. Roco, Optimal low symmetric dissipation Carnot engines and refrigerators, Phys. Rev. E 85 (2012) 010104(R), DOI:10.1103/PhysRevE.85.010104

[37] Y. Wang, M. Li, Z. C. Tu, A. Calvo Hernández, J. M. M. Roco, Coefficient of performance at maximum figure of merit and its bounds for low-dissipation Carnot-like refrigerators, Phys. Rev. E 86 (2012) 011127 DOI:10.1103/PhysRevE.86.011127

[38] Y. Hu, F. Wu, Y. Ma, J. He, J. Wang, A. Calvo Hernández, J. M. M. Roco, Coefficient of performance for a low-dissipation Carnot-like refrigerator with nonadiabatic dissipation, Phys. Rev. E 88 (2013) 062115, DOI:10.1103/PhysRevE.88.062115

[39] V. Holubec, R. Artem, Efficiency at and near maximum power of low-dissipation heat engines Phys. Rev. E 92 
(2015) 052125 DOI:10.1103/PhysRevE.92.052125

[40] J. Gonzalez-Ayala, A. Calvo Hernández, J. M. M. Roco, Irreversible and endoreversible behaviors of the LD-model for heat devices: the role of the timeconstraints and symmetries on the performance at maximum $\chi$ figure of merit. J . Stat. Mech. (2016) 073202, DOI:10.1088/1742-5468/2016/07/073202

[41] T. Schmiedl, U. Seifert, Efficiency at maximum power: An analytically solvable model for stochastic heat engines, EPL 92 (2008) 20003, DOI:10.1209/0295$5075 / 81 / 20003$

[42] V. Holubec, An exactly solvable model of a stochastic heat engine: optimization of power, power fluctuations and efficiency, J. Stat. Mech. (2014) P05022, DOI:10.1088/1742-5468/2014/05/P05022

[43] P. R. Zulkowski, M. R. DeWeese, Optimal protocols for slowly driven quantum systems, Phys. Rev. E 92 (2015) 032113 , DOI:10.1103/PhysRevE.92.032113

[44] J. Gonzalez-Ayala, J. M. M. Roco, A. Medina, A. Calvo Hernández, Carnot-like heat engines versus low-dissipation models, Entropy 19 (2017) 182, DOI:10.3390/e19040182

[45] R. S. Johal, Heat engines at optimal power: Lowdissipation versus endoreversible model Phys. Rev. E 96 (2017) 012151, DOI:10.1103/PhysRevE.96.012151

[46] F. Angulo-Brown, An ecological optimization criterion for finite-time heat engines J. Appl. Phys. 69 (1991) 7465-7469, DOI:10.1063/1.347562

[47] S. Velasco, J. M. M. Roco, A. Medina, A. Calvo Hernández, Irreversible refrigerators under per-unit-time coefficient of performance optimization, Appl. Phys. Lett. 71 (1997) 01130-1132, DOI:10.1063/1.120437

[48] J. Guo, J. Wang, Y. Wang, J. Chen, Universal efficiency bounds of weak-dissipative thermodynamic cycles at the maximum power output, Phys. Rev. E 87 (2013) 012133 DOI:10.1103/PhysRevE.87.012133

[49] E. Açıkkalp, Exergetic sustainability evaluation of irreversible Carnot refrigerator Physica A: Statistical Mechanics and its Applications 436 (2015) 311-320, DOI:10.1016/j.physa.2015.04.036

[50] B. Cleuren, B. Rutten, C. Van den Broeck, Universality of efficiency at maximum power: Macroscopic manifestation of microscopic constraints, Eur. Phys. J. Special Topics 224 (2015) 879, DOI:10.1140/epjst/e2015-024338

[51] Y. Zhang, J. Guo, G. Lin, J. Chen, Universal Optimization Efficiency for Nonlinear Irreversible Heat Engines,
Journal of Non-Equilibrium Thermodynamics 42 (2017) 253-263, DOI:10.1515/jnet-2016-0065

[52] A. Calvo Hernández, A. Medina, J. M. M. Roco, J. A. White, S. Velasco, Unified optimization criterion for energy converters, Phys. Rev. E 63 (2001) 037102, DOI:10.1103/PhysRevE.63.037102

[53] C. de Tomás, J. M. M. Roco, A. Calvo Hernández, Y. Wang, Z. C. Tu, Low-dissipation heat devices: Unified trade-off optimization and bounds, Phys. Rev. E $\mathbf{8 7}$ (2013) 012105, DOI:10.1103/PhysRevE.87.012105

[54] Y. Yuan, R. Wang, J. He, Y. Ma, J. Wang, Coefficient of performance under maximum $\chi$ criterion in a two-level atomic system as a refrigerator, Phys. Rev. E 90 (2014) 052151, DOI:10.1103/PhysRevE.90.052151

[55] S. Sheng, P. Yang, Z. C. Tu, Coefficient of Performance at Maximum $\chi$-Criterion for Feynman Ratchet as a Refrigerator, Commun. Theor. Phys. 62 (2014) 589-595, DOI:10.1088/0253-6102/62/4/16

[56] R. Long, W. Liu, Coefficient of performance and its bounds with the figure of merit of a general refrigerator Phys Scr 90 (2015) 025207, DOI:10.1088/00318949/90/2/025207

[57] Y. Zhang, C. Huang, G. Lin, J. Chen, Universality of efficiency at unified trade-off optimization Phys. Rev. E 93 (2016) 032152, DOI:10.1103/PhysRevE.93.032152

[58] C. Lu, L. Bai, Nonlinear Dissipation Heat Devices in Finite-Time Thermodynamics: An Analysis of the TradeOff Optimization, Journal of Non-Equilibrium Thermodynamics 42 (2017) 277-286, DOI:10.1515/jnet-20160071

[59] M. H. Rubin, Optimal configuration of a class of irreversible heat engines. I, Phys. Rev. A 19 (1979) 1272, DOI:10.1103/PhysRevA.19.1272.

[60] A. Calvo Hernández, A. Medina, J. M. M. Roco, Time, entropy generation, and optimization in lowdissipation heat devices, New J Phys 17 (2015) 075011, DOI:10.1088/1367-2630/17/7/075011; Corrigendum New J Phys 18 (2016) 019501, DOI:10.1088/13672630/18/1/019501

[61] J. Gonzalez-Ayala, L. A. Arias-Hernández and F. Angulo-Brown, A graphic approach to include dissipative-like effects in reversible thermal cycles, Eur. Phys. J. B 90 (2017) 86, DOI:10.1140/epjb/e201780001-4 